CLINICAL STUDY

\title{
Efficacy of imatinib mesylate in advanced medullary thyroid carcinoma
}

\author{
Karin Frank-Raue, Michael Fabel ${ }^{1}$, Stefan Delorme ${ }^{1}$, Uwe Haberkorn ${ }^{2}$ and Friedhelm Raue \\ Endocrine Practice, Brückenstr. 21, 69120 Heidelberg, Germany, ${ }^{1}$ Department of Radiology, German Cancer Centre, 69120 Heidelberg, Germany and \\ ${ }^{2}$ Department of Nuclear Medicine, University of Heidelberg, 69120 Heidelberg, Germany \\ (Correspondence should be addressed to K Frank-Raue; Email: karin.frankraue@raue-endokrinologie.de)
}

\begin{abstract}
Objective: Medullary thyroid carcinoma (MTC) is often associated with gain-of-function mutations in the RET proto-oncogene, which is found in all hereditary cases and most sporadic cases. The activated RET receptor tyrosine kinase can be inhibited by tyrosine kinase inhibitors in vitro. We evaluated the efficacy of treatment with imatinib mesylate, a tyrosine kinase inhibitor, in patients with advanced MTC.

Design and patients: In this open-label clinical trial, nine patients, eight with sporadic and one with hereditary MTC, with unresectable, measurable, progressive metastases were treated with imatinib mesylate $600 \mathrm{mg}$ daily. The tumour response to imatinib was evaluated after 3, 6 and 12 months by computed tomography and after 1 month by ${ }^{18}$ F-fluoro-2-deoxy D-glucose position-emission tomographic scanning. The median duration of therapy was 8 months.

Results: Overall, stable disease occurred in five patients for up to 6 months and in one patient for up to 12 months, with a median duration of progression-free survival of 6 months. Four patients had progressive disease after 12 months. One patient stopped therapy after 2 weeks because of worsening of diarrhoea. Therapy was well tolerated, although transient mild-to-moderate nausea $(n=3)$, oedema $(n=3)$, diarrhoea $(n=2)$ and skin rash $(n=2)$ were observed.

Conclusion: Imatinib mesylate is well tolerated, no tumour remission was observed, only transient stable disease was achieved in some patients with advanced MTC.

European Journal of Endocrinology 157 215-220
\end{abstract}

\section{Introduction}

Medullary thyroid carcinoma (MTC) is sporadic in $75 \%$ and hereditary in $25 \%$ of all cases. The familial variety of MTC is inherited as an autosomal dominant trait and is associated with the multiple endocrine neoplasia (MEN) type 2 syndrome. Germline mutations in RET proto-oncogene cause the inheritance of the MEN2 syndrome, but somatic mutations in the RET gene have been found in many sporadic cases of MTC $(1,2)$. The RET gene encodes a transmembrane tyrosine kinase receptor, which is constitutively activated by the mutations observed in MEN2, and is involved in the pathogenesis of MTC. Prophylactic surgery in carriers of RET mutations has significantly improved the prognosis of MEN2 patients (3). Patients with sporadic MTC, however, are often incurable because the cancer is not diagnosed until after it has metastasised. Systemic therapies like chemotherapy have not been effective in treating this disease (4). Recently, various kinds of therapeutic approaches such as tyrosine kinase inhibition, gene therapy, monoclonal antibodies against oncogene products, and nuclease-resistant aptamers that recognise and inhibit RET have been developed (5).
Targeted therapies, such as tyrosine kinase inhibitors, are used in chronic myelogenous leukaemia (CML) and gastrointestinal stromal tumours (GIST) with good results (6-8). RET appears to be an excellent target for tyrosine kinase inhibitors like STI 571, ZD6474 and BAY 43-9006 (9), some of which are also effective inhibitors of vascular endothelial growth factor receptor 2. The growth of an MTC cell line (TT, human MTC cell line with a cys634trp heterozygous mutation) has been inhibited by different tyrosine kinase inhibitors in vitro and in an in vivo xenograft model (10-12). Imatinib (STI 571) inhibited the RET phosphorylation in two MTCderived cell lines in a dose-dependent manner (12). However, the $\mathrm{IC}_{50}$, the concentration that causes $50 \%$ growth inhibition of imatinib necessary to inhibit RET in vitro is high when compared with other smallmolecule kinase inhibitors of RET activity $(5,9)$. In 2002/2003, when this study was initiated, imatinib was the only substance to be used in humans with CML and GIST (6-8).

In this study, we treated nine patients with advanced, progressive MTC with imatinib mesylate to evaluate its activity in this tumour type. 


\section{Subjects and methods}

The study was approved by the institutional review board of the University of Heidelberg (no. L-331/2003) and written informed consent was obtained from all patients before they were enrolled in the study. In this preliminary study, a maximum of 10 patients was planned to be included.

\section{Patients}

Nine patients aged more than 18 years with a histologically confirmed diagnosis of metastatic MTC that was progressive and unresectable were eligible for the study. Criteria for inclusion were at least one measurable lesion, adequate performance status (Eastern Cooperative Oncology Group grade 0-3), and adequate hepatic and renal function.

\section{Study design}

An open-label clinical trial was designed to evaluate the activity of imatinib mesylate inducing objective responses in metastatic MTC. The primary end point of this study was evidence of anti-neoplastic activity as measured by objective tumour response. The secondary efficacy parameters were changes in metabolic profiles assessed by ${ }^{18} \mathrm{~F}$-fluoro-2-deoxy D-glucose position-emission tomographic scanning $\left({ }^{18}\right.$ F-FDG PET $)$, changes in the tumour markers calcitonin and carcinoembryonic antigen (CEA) and safety and tolerability assessments. Patients received imatinib mesylate $600 \mathrm{mg} /$ day orally as three $100 \mathrm{mg}$ capsules taken twice daily with food. Patients were treated for up to 12 months. Treatment after completion of the study was at the discretion of the investigator. Patients had regular physical examinations, evaluation of their performance status, and complete blood chemistry at baseline, 1 month, 3 months and every 3 months thereafter. The study was designed by the principal investigator in collaboration with Novartis.

\section{Assessment of response evaluation and toxicity}

Tumour responses were evaluated by computed tomography after 3 months, 6 months and then every 6 months afterward. Assessments were performed according to the RECIST standard and based solely on computed tomography (13). The sum of the longest unidimensional diameter of up to 10 lesions was selected at baseline to be measured throughout the study. Responses were classified as follows: a complete response (CR) was the disappearance of all target lesions; a partial response (PR) was a decrease to $<30 \%$ of the sum of the longest diameters of the target lesions when compared with the sum of the longest diameter at baseline; stable disease (SD) was classified as meeting the requirements of neither PR nor progressive disease (PD); PD was defined as a $20 \%$ or more increase in the sum of the longest diameters of the target lesions when compared with the baseline assessment.

The ${ }^{18}$ F-FDG PET scanning was performed at baseline and after 1 month of treatment to assess changes in the metabolic profile of the tumour. Standard uptake values 'Baseline (SUV)' refers to the PET value prior to therapy. The reproducibility of serial PET measurements has been studied by Weber et al. showing a s.D. of the mean percentage difference of about $5-10 \%$. Thus, for a 'typical' lesion, changes of a parameter of more than $20 \%$ are outside the $95 \%$ range for spontaneous fluctuations, and therefore can be considered to reflect true changes in glucose metabolism of the tumour mass (14). In analogy to the RECIST criteria for morphologic imaging, the sum of up to 10 accumulating lesions was compared prior with and during therapy. This strategy assumes that FDG uptake is mainly a marker of tumour viability. However, a decrease in the glucose metabolism in viable cells caused by the drug cannot be excluded and may contribute to changes in FDG uptake. The sum of SUV of up to 10 active lesions were compared, and the response was classified as a PR when there was a $15 \%$ decrease in the sum of SUV, or as PD when there was an increase of more than $25 \%$ in the sum of SUV or new lesions, SD and as when there was neither PD nor PR (15).

The following criteria were used to define clinical response for calcitonin and CEA levels: CR was a decrease to undetectable levels; PR was a $25 \%$ or more decrease in serum tumour marker levels observed after 1 month, and then at 3-month intervals; PD was an increase of $25 \%$ or more; and SD was neither an increase to quality for PD nor a sufficient decrease to quality for PR. Calcitonin determination was done by chemiluminescence assay (Nichols Institut), normal range below $10 \mathrm{pg} / \mathrm{ml}$. CEA determination was also done by chemiluminescence assay (Bayer Health Care), with a normal range below $5 \mathrm{ng} / \mathrm{ml}$.

Safety assessments included an evaluation of adverse events, laboratory parameters, physical examinations and performance status. Toxic effects were graded according to the National Cancer Institute Common Toxicity Criteria, version 2 (16).

In all patients, germline RET mutation analysis had been performed as previously reported (17).

\section{Results}

\section{Patients}

Between February 2004 and December 2005, nine patients with advanced, metastatic MTC were included in this study (Table 1). Eight patients had sporadic MTC with no germline mutation in the RET proto-oncogene, and one patient suffered from MEN2B with a typical mutation at codon 918 (patient no 6). In all cases, previous therapy included thyroidectomy and cervical lymph node dissection. Octreotide therapy in three 
Table 1 Patient characteristics $(n=9)$.

\begin{tabular}{lc}
\hline Age (years) & 59 \\
Median & $19-68$ \\
Range & 7 \\
Sex (n) & 2 \\
Male & \\
Female & 1 \\
Performance status ECOG (n) & 6 \\
Grade 0 & 2 \\
Grade 1 & 9 \\
Grade 2 & \\
Time from diagnosis to start of & 9 \\
therapy (years, median) & 7 \\
Site of metastases (n) & 5 \\
Local (cervical, mediastinal) & 3 \\
Lung & \\
Liver & \\
Bone & 30700 \\
Tumour markers, pre-treatment & $6200-104692$ \\
Calcitonin (pg/ml) & \\
Median & 254 \\
Range & $20-590$ \\
CEA (ng/ml) & \\
Median & \\
Range & \\
\hline
\end{tabular}

patients had been stopped at least 3 months prior to the beginning of this study. The most common metastatic sites were the cervical lymph nodes $(n=9)$, lung $(n=7)$, liver $(n=5)$ and bone $(n=3)$. In all patients, the disease was progressive with growth of metastases $(9-82 \%$ increase in tumour mass, RECIST criteria) within the past 12 months (Table 2), and a doubling time of tumour marker levels of 6 months to 4 years (median 1.25 years; Tables 3 and 4 ) before beginning imatinib mesylate treatment.

\section{Treatment}

The follow-up of all patients ranged from 3 to 28 months (median 14 months), and the duration of imatinib mesylate administration ranged from 0.5 to 28 months (median 13 months). Five patients completed 1 year of treatment with imatinib mesylate. One patient died 4 months after initiation of therapy because of tumour cachexia and respiratory problems (patient no. 2). Another patient died after 7 months because of a metabolic disturbance (patient no. 9). No sign of tumour progression was observed in either patient. In another patient, therapy was stopped after 2 weeks because of worsening diarrhoea, and in an additional patient after 3 months because of progressive disease. Four patients continued to take imatinib for an additional 5-16 months and are still alive. Two patients died 7 (patient no. 4) and 17 (patient no. 7) months after termination of the study.

\section{Tumour response}

The objective tumour response determined by $\mathrm{CT}$ is shown in Table 2. Because one patient discontinued treatment after two weeks due to diarrhoea, eight patients were evaluated for tumour assessment and had at least the first post-baseline assessment with computed tomography after 3 months. A complete or partial response was not seen. After 3 months of treatment, seven patients had stable disease and one patient had progressive disease and discontinued treatment. After 6 months, five out of the six patients were stable; one patient progressed, but remained on treatment. After 12 months, one patient was stable and four patients had documented progression. Overall, two patients had

Table 2 Objective responses according to the RECIST criteria (computerised tomography) to imatinib mesylate in patients with advanced medullary thyroid carcinoma, percentage of the sum of longest diameter, differences against baseline.

\begin{tabular}{|c|c|c|c|c|c|c|}
\hline \multirow[b]{2}{*}{ Patient no. } & \multirow[b]{2}{*}{3 months } & \multirow[b]{2}{*}{6 months } & \multirow[b]{2}{*}{12 months } & \multirow[b]{2}{*}{$\begin{array}{c}\text { All over } \\
\text { response after } 1 \\
\text { year }\end{array}$} & \multicolumn{2}{|c|}{ Tumour growth in $\%$ within } \\
\hline & & & & & $\begin{array}{l}1 \text { year before } \\
\text { imatinib }\end{array}$ & $\begin{array}{c}1 \text { year after study } \\
\text { interval }\end{array}$ \\
\hline 1 & +5 & +5 & +10 & $\begin{array}{l}\text { SD still after } 28 \\
\text { months }\end{array}$ & +9 & $+10^{\mathrm{a}}$ \\
\hline 2 & +18 & - & - & $\begin{array}{l}\text { Patient died after } \\
4 \text { months }\end{array}$ & +43 & - \\
\hline $\begin{array}{l}3 \\
4\end{array}$ & $\begin{array}{r}+5 \\
+16\end{array}$ & $\begin{array}{l}+14 \\
+41\end{array}$ & $\begin{array}{l}+23 \\
+44\end{array}$ & $\begin{array}{l}\text { PD } \\
\text { PD }\end{array}$ & $\begin{array}{l}+57 \\
+79\end{array}$ & $+6^{\mathrm{a}}$ \\
\hline 5 & - & - & $\begin{array}{l}+44 \\
-\end{array}$ & $\begin{array}{l}\text { Stopped after } 2 \\
\text { weeks }\end{array}$ & - & - \\
\hline 6 & +2 & +13 & +26 & PD & +14 & $+18^{\mathrm{a}}$ \\
\hline 7 & +32 & - & - & $\begin{array}{l}\text { Changed to che- } \\
\text { motherapy }\end{array}$ & +82 & - \\
\hline 8 & +5 & +5 & +32 & PD & +23 & $+16^{a}$ \\
\hline 9 & +5 & +6 & - & $\begin{array}{l}\text { Patient died after } \\
7 \text { months }\end{array}$ & - & - \\
\hline \multicolumn{7}{|c|}{ Summary of results } \\
\hline SD & 7 & 5 & 1 & & & \\
\hline PD & 1 & 1 & 4 & & & \\
\hline
\end{tabular}

PD, progressive disease $(+20 \%)$; SD, stable disease $(+20 \%$ to $-30 \%)$.

${ }^{a}$ Continuation of imatinib mesylate therapy after 1 year. 
Table 3 Changes in plasma calcitonin during imatinib mesylate treatment expressed as a percentage of baseline calcitonin (progressive disease (PD), $>25 \%$; stable disease (SD), +25 to $-25 \%$; partial response (PR), $<-25 \%$ ).

\begin{tabular}{|c|c|c|c|c|c|c|c|}
\hline Patient no. & 1 month & 3 months & 6 months & 9 months & 12 months & $\begin{array}{c}\text { All over } \\
\text { response after } \\
1 \text { year }\end{array}$ & $\begin{array}{c}\text { Pre-imatinib } \\
\text { doubling time } \\
\text { (years) }\end{array}$ \\
\hline 1 & +60 & +100 & +80 & +103 & +81 & PD & 2 \\
\hline 2 & +5 & -1 & - & - & - & & 0.5 \\
\hline 3 & -22 & +30 & +97 & +54 & +118 & PD & 4 \\
\hline 4 & +61 & +61 & - & +86 & +102 & PD & 0.75 \\
\hline 5 & - & - & - & - & - & & \\
\hline 6 & +14 & +19 & +37 & +18 & +95 & SD & 1 \\
\hline 7 & -38 & -8 & - & - & - & & 1.25 \\
\hline 8 & -37 & -14 & +1 & -35 & -5 & SD & 1.5 \\
\hline 9 & -48 & +11 & -16 & - & - & - & \\
\hline \multicolumn{8}{|c|}{ Summary of results } \\
\hline PR & 3 & - & - & 1 & - & & \\
\hline SD & 3 & 5 & 2 & 1 & 1 & & \\
\hline PD & 2 & 3 & 3 & 3 & 4 & & \\
\hline
\end{tabular}

stable disease for 3 months, four for 6 months and one for 12 months. The patient taking imatinib mesylate for more than 12 months (patient no. 1) has experienced durable stable disease (by CT) for up to 28 months. This patient with the prolonged stable disease had also the lowest pre-therapeutic tumour growth. The median progression-free survival was 6 months. In one patient, progressive disease was seen after 3 months, in one patient after 6 months, and in three patients after 12 months of imatinib treatment.

The ${ }^{18} \mathrm{~F}$-FDG PET proved to be a reliable indicator of tumour activity (Table 5). After 1 month of treatment, three patients showed decreased glucose uptake (SUV -17 to $-48 \%$ ), one patient progressed (SUV $+28 \%$ ), and in all others, stable disease could be documented.

The tumour marker calcitonin showed a shortlasting decrease (PR) in three patients after 1 month of treatment, but SD was observed in only one patient after 1 year, and PD in four patients (Table 3). After 1 year, CEA increases (PD) were seen in three patients and SD in two patients (Table 4).
The ECOG performance status was stable in most patients throughout therapy $(n=7)$, but performance status decreased in two patients with PD.

Treatment with imatinib mesylate was generally well tolerated. No grade 3 or 4 toxicities were observed. Treatment was stopped after 2 weeks in one patient because of worsening of pre-existing diarrhoea, and two short treatment interruptions occurred ( 3 days to 1 week) because of skin rash. Three patients had vomiting and abdominal pain, two had diarrhoea, and three had oedema, especially periorbitally.

\section{Discussion}

This open-label clinical study demonstrated that imatinib mesylate seems to be active in some patients with advanced, progressive MTC. Stable disease was achieved by seven out of eight patients after 3 months, but four patients progressed within 12 months and two deaths occurred. In one patient who continued the

Table 4 Changes in serum CEA levels during imatinib mesylate treatment expressed as a percentage of baseline CEA (progressive disease (PD), $>25 \%$; stable disease (SD), +25 to $-25 \%$; partial response, $<-25 \%$ ).

\begin{tabular}{|c|c|c|c|c|c|c|c|}
\hline Patient no. & 1 month & 3 months & 6 months & 9 months & 12 months & $\begin{array}{c}\text { All over } \\
\text { response after } \\
1 \text { year }\end{array}$ & $\begin{array}{c}\text { Pre-imatinib } \\
\text { doubling time } \\
\text { (years) }\end{array}$ \\
\hline 1 & -5 & -19 & +80 & -15 & -21 & SD & 2 \\
\hline 2 & +65 & +266 & - & - & - & & 0.5 \\
\hline 3 & +6 & -1 & -19 & -15 & +4 & SD & 4 \\
\hline 4 & +29 & +213 & - & +290 & +671 & PD & 0.75 \\
\hline 5 & - & - & - & - & - & & \\
\hline 6 & +16 & +57 & +27 & +69 & +74 & PD & 1 \\
\hline 7 & +35 & +8 & - & - & - & & 0.2 \\
\hline 8 & +45 & +60 & +53 & +51 & +92 & PD & 1.5 \\
\hline 9 & +9 & +29 & - & - & - & - & \\
\hline \multicolumn{8}{|c|}{ Summary of results } \\
\hline SD & 4 & 3 & 1 & 2 & 2 & & \\
\hline PD & 4 & 5 & 3 & 3 & 3 & & \\
\hline
\end{tabular}


Table 5 Positron-emission tomography comparing baseline to uptake after one month of imatinib mesylate treatment (percentage of differences of the sum of standard uptake values; progressive disease (PD), $>25 \%$ or new lesion; stable disease (SD), +25 to $-15 \%$; partial response (PR), $<-15 \%$; EORTC-criteria).

\begin{tabular}{lrc}
\hline Patient no. & 1 month & Comment \\
\hline 1 & +28 & $\mathrm{PD}$ \\
2 & 0 & $\mathrm{SD}$ \\
3 & -12 & $\mathrm{SD}$ \\
4 & +5 & $\mathrm{SD}$ \\
5 & - & - \\
6 & -19 & $\mathrm{PR}$ \\
7 & +8 & $\mathrm{SD}$ \\
8 & -48 & $\mathrm{PR}$ \\
9 & -17 & $\mathrm{PR}$ \\
Summary of results & & \\
PR & 3 & \\
SD & 4 & \\
PD & 1 & \\
\hline
\end{tabular}

imatinib mesylate therapy, stable disease lasted for 28 months, but this patient with the prolonged stable disease also had the lowest tumour growth before initiation of imatinib mesylate. A rapid decline in the standardised uptake values of ${ }^{18} \mathrm{~F}$-FDG in tumour tissue after 1 month of treatment with imatinib mesylate was seen in three patients by PET scanning correlating to the decline in calcitonin levels. The initial decline in the tumour marker calcitonin might reflect an inhibition of secretion or synthesis without any effect on tumour mass. In patient no 8, during follow-up RECIST criteria showed progressive disease despite an early decrease in SUV and calcitonin level. After 1 year of treatment, only a few patients had stable disease as documented in all the parameters. Definition or documentation of response in slow growing tumours is difficult, especially in MTC were metastases often contain a large amount of amyloid or calcifications. This has to be taken into account when planning therapeutic studies.

MTC is a highly differentiated, slow growing tumour in most cases. Therefore, it is important to document the long-term outcome in order to show the beneficial effects of treatment. Despite extensive metastatic disease and local recurrence, only two patients died at 4 and 7 months after initiating therapy, because of respiratory problems due to local and lung metastases and metabolic disturbance respectively. These results are consistent with a preliminary study done by Zonenberg et al. (18), who also observed long-term stable disease with imatinib mesylate treatment over 18 months in 4 out of 15 patients with advanced MTC. No remission of the tumour was observed. In another preliminary phase 2 study using imatinib mesylate in 15 patients with advanced endocrine tumours, disease progression was seen in four out of six patients with MTC (19).

The standard procedure for MTC is surgical resection of the tumour and local metastases, although in many cases, this procedure is not curative and additional metastectomies do not improve survival. Current systemic therapies, especially in advanced tumour stages, such as chemotherapy or octreotide, are ineffective. New targeted therapies, which disrupt signalling pathways inappropriately activated in cancer cells, leaving normal cells relatively unscathed, like protein kinase inhibitors, offer new therapeutic opportunities $(8,9)$. One of these targeted therapies is imatinib mesylate, a tyrosine kinase inhibitor, which has become the standard treatment for chronic myelogenous leukaemia and GIST $(6,7,20)$.

Achievement of transient SD (median 6 months) in our patients cannot be definitively attributed to imatinib mesylate, given the single-arm study design, the small series and the relatively slow-growing nature of this disease. In patients with sporadic MTC, only 40-60\% somatic RET mutations are found, this might be one explanation for reduced effectiveness of tyrosine kinase inhibition therapy. Although no remissions were observed, some patients had durable evidence of a stable disease that lasted more than 6 months with few side effects. The other patients may have developed early resistance to imatinib mesylate. An explanation may be that in advanced MTC multistep carcinogenesis may make tumours independent of the initial germline/somatic RET mutation. Other molecular mechanisms of resistance in these patients have to be discussed. It is not clear if imatinib acts directly on RET tyrosine kinase, which is involved in tumour development in MEN2 patients and might be responsible in some respect for tumour genesis in sporadic MTC or other downstream intracellular mechanisms. Suppression of RET activity arrests the growth of MTC cells in vitro and in human MTC cell xenografts (11) by inhibition of the wild-type enzyme as well as most of the mutant, activated forms of RET. Interestingly, RET proteins carrying mutations at residue V804 are resistant to the tyrosine kinase inhibitor ZD6474, and this residue seems to have a gate-keeper function (21). V804 mutations are present alone or with other RET mutations in hereditary as well as somatic mutations in sporadic cases. Selection of cells harbouring a RET mutation resistant to tyrosine kinase treatment might be an explanation for primary resistance in MTC. Another explanation for the relatively poor outcome may be that the dose of imatinib mesylate was too low to inhibit RET activity or phosphorylation, as suspected from in vitro studies (12). In comparison to other small-molecule kinase inhibitors of RET activity, imatinib exerts a low inhibition effect. The in vitro $\mathrm{IC}_{50}$ concentration required to inhibit RET activity is $10 \mu \mathrm{M}$ compared with e.g. $100 \mathrm{nM}$ using ZD6474 (9).

The power of this preliminary study is limited by the small number of patients, the rareness of the disease and the slow growing nature of the tumour. This small study allows no final conclusion. Careful studies with more potent or specific RET tyrosine kinase inhibitors as well as better molecularly defined patients for treatment are necessary for improving the anti-tumour effect. Estimating the benefits of treatment in terms of stable disease or 
progression-free survival requires large multicentric randomised trials versus placebo and patients should be included in prospective multicentre trials that are in progress.

Overall, imatinib was well tolerated with side effects similar to those reported in large populations of chronic myeloid leukaemia and GIST patients. In contrast to CML, myelotoxicity was not observed. The most significant adverse effects were gastrointestinal problems such as diarrhoea, vomiting and abdominal pain, and facial oedema, especially periorbital oedema. Most of these adverse effects were of short duration and not severe. Since diarrhoea is one of the clinical signs of advanced disease in MTC, it is difficult to differentiate between the toxic effects of imatinib and disease symptoms. Three patients had no side effects from imatinib treatment.

The current findings suggest that new targeted therapies with tyrosine kinase inhibitors like imatinib might be a good way to overcome the therapeutic nihilism in advanced MTC with tolerable side effects, but careful study of molecular mechanisms will be needed in order to reinforce the anti-tumour activity.

Note added in proof Similar results have been found by de Groot et al. (Journal of Clinical Endocrinology and Metabolism, Epub ahead of print).

\section{Acknowledgement}

We thank Dr G Middeler, Diapharmgruppe, Lübeck, Germany, and Dr Ch Hosius, Novartis, Nürnberg, Germany, for their expert advice. This study was funded by a grant from Novartis, Nürnberg, Germany.

\section{References}

1 Eng C, Mulligan LM, Healey CS, Houghton C, Frilling A, Raue F, Thomas GA \& Ponder BAJ. Heterogeneous mutation of the RET proto-oncogene in subpopulations of medullary thyroid carcinoma. Cancer Research 199656 2167-2170.

2 Schilling T, Bürck J, Sinn HP, Clemens A, Otto HF, Höppner W, Herfarth C, Ziegler R, Schwab M \& Raue F. Prognostic value of codon 918 (ATG-ACG) RET proto-oncogene mutations in sporadic medullary thyroid carcinoma. International Journal of Cancer 2001 95 62-66.

3 Machens A, Nicolli-Sire P, Hoegel J, Frank-Raue K, van Vroonhoven TJ, Roeher HD, Wahl RA, Lamesch P, Raue F, ConteDevolx B \& Dralle H. Early malignant progression of hereditary medullary thyroid cancer. New England Journal of Medicine 2003 349 1517-1525.

4 Nocera M, Baudin E, Pellegriti G, Cailleux AF, Mechelany-Corone C \& Schlumberger M. Treatment of advanced medullary thyroid cancer with an alternating combination of doxorubicin-streptozocin and 5 FU-dacarbazine. Groupe d'Etude des Tumeurs a Calcitonine (GETC). British Journal of Cancer 200083 715-718.

5 de Groot JWB, Links TP, Plukker JTM, Lips CJM \& Hofstra RMW. RET as a diagnostic and therapeutic target in sporadic and hereditary endocrine tumours. Endocrine Reviews 200627 535-560.

6 Druker BJ, Talpaz M, Resta DJ, Peng B, Buchdunger E, Ford JM, Lydon NB, Kantarjian H, Capdeville R, Ohno-Jones S \&
Sawyers CL. Efficacy and safety of a specific inhibitor of the BCRABL tyrosine kinase in chronic myeloid leukemia. New England Journal of Medicine 2001344 1031-1037.

7 Demetri GD, von Mehren M, Blanke CD, Van den Abbeele AD, Eisenberg B, Roberts PJ, Heinrich MC, Tuveson DA, Singer S, Janicek M, Fletcher JA, Silverman SG, Silberman SL, Capdeville R, Kiese B, Peng B, Dimitrijevic S, Druker BJ, Corless C, Fletcher CD \& Joensuu H. Efficacy and safety of imatinib mesylate in advanced gastrointestinal stromal tumors. New England Journal of Medicine $2002347472-480$.

8 Krause DS \& Van Etten RA. Tyrosine kinases as targets for thyroid cancer therapy. New England Journal of Medicine 2005353 172-187.

9 Santoro M \& Carlomagno F. Drug insight: small-molecule inhibitors of protein kinases in the treatment of thyroid cancer. Nature Clinical Practice. Endocrinology \& Metabolism 20062 42-52.

10 Cohen MS, Hussain HB \& Moley JF. Inhibition of medullary thyroid carcinoma cell proliferation and RET phosphorylation by tyrosine kinase inhibitors. Surgery 2002132 960-966.

11 Strock CJ, Park JI, Rosen DM, Ruggeri B, Denmeade SR, Ball DW \& Nelkin BD. Activity of irinotecan and the tyrosine kinase inhibitor CEP-751 in medullary thyroid cancer. Journal of Clinical Endocrinology and Metabolism 200691 79-84.

12 de Groot JW, Plaza Menacho I, Schepers H, Drenth-Diephuis LJ, Osinga J, Plukker JT, Links TP, Eggen BJ \& Hofstra RM. Cellular effects of imatinib on medullary thyroid cancer cells harboring multiple endocrine neoplasia type $2 \mathrm{~A}$ and $2 \mathrm{~B}$ associated RET mutations. Surgery $2006139806-814$.

13 Therasse P, Arbruck SG, Eisenhauer EA, Wanders J, Kaplan RS, Rubinstein L, Verweij J, Van Glabbeke M, Van Oosterom AT, Christian MC \& Gwyther SG. New guidelines to evaluate the response to treatment in solid tumors. Journal of the National Cancer Institute 200092 205-216.

14 Weber WA, Ziegler SJ, Thödtmann R, Hanauske A-R \& Schwaiger M. Reproducibility of metabolic measurements in malignant tumours using FDG PET. Journal of Nuclear Medicine 199940 1771-1777.

15 Young H, Baum R, Cremerius U, Herholz K, Hoekstra O, Lammertsma AA, Pruim J \& Price P. Measurement of clinical and subclinical tumour response using 18F-fluorodeoxyglucose and position emission tomography: review and 1999 EORTC recommendations. EORTC PET Study Group. European Journal of Cancer 199935 1773-1782.

16 Cancer therapy evaluation program. Common toxicity criteria manual: common toxicity criteria, version 2.0. Bethesda, MD: National Cancer Institute, 1999. (Also available at http://ctep. cancer.gov/forms/CTCManual-v4-10-4-99.pdf).

17 Frank-Raue K, Rondot S, Höppner W, Goretzki P, Raue F \& Meng W. Coincidence of multiple endocrine neoplasia type 1 and 2: mutations in the RET proto-oncogene and MEN 1 tumor suppressor gene in a family presenting with recurrent primary hyperparathyroidism. Journal of Clinical Endocrinology and Metabolism $2005904063-4067$.

18 Zonnenberg BA, Links T, Quarles van Uffort P, Lips C, De Groot J \& Voest E. Phase-2 study of imatinib in metastasized medullary thyroid carcinoma. Journal of Clinical Oncology $2006 \mathbf{2 4} 15550$.

19 Gross DJ, Munter G, Bitan M, Siegal T, Gabizon A, Weitzen R, Merimsky O, Ackerstein A, Salmon A, Sella A \& Slavin S. The role of imatinib mesylate (Glivec) for treatment of patients with malignant endocrine tumors positive for c-kit or PDGF-R. Endocrine-Related Cancer 200613 535-540.

20 Savage DG \& Antman KH. Imatinib mesylate - a new oral targeted therapy. New England Journal of Medicine 2002346 683-693.

21 Carlomagno F, Guida T, Anaganti S, Vecchio G, Fusco A, Ryan AJ, Billaud M \& Santoro M. Disease associated mutations at valine 804 in the RET receptor tyrosine kinase confer resistance to selective kinase inhibitors. Oncogene 200423 6056-6063.

Received 21 November 2006

Accepted 16 May 2007 